\title{
Topiramate-Related Hyperammonemia
}

\author{
Kelly Moore, PharmD', Deepali Dixit, PharmD, BCPS ${ }^{2,3}$, \\ and Mary Wagner, PharmD, BCPS ${ }^{2,3}$
}

Journal of Pharmacy Technology

2016, Vol. 32(I) 34-36

(C) The Author(s) 2015

Reprints and permissions:

sagepub.com/journalsPermissions.nav

DOI: |0.1 |77/8755|225|5605849

pharmatech.sagepub.com

(A)AGE

\begin{abstract}
Objective: To report a case of hyperammonemia associated with the interaction between topiramate and valproic acid. Case Summary: We present a patient case with topiramate-related hyperammonemia. The patient was on topiramate prior to admission and presented with an elevated ammonia level following 2 doses of valproic acid. The increased dosing frequency of valproic acid further exacerbated the hyperammonemia. On discontinuation of topiramate and the tapering of the dose of valproic acid the patient's ammonia level returned to normal. Discussion: Valproic acid-induced hyperammonemic encephalopathy is well documented and there are several proposed mechanisms. The interaction between valproic acid and topiramate causing drug-induced hyperammonemia encephalopathy is documented in several case reports. The interaction is associated with the addition of topiramate to a patient's medication regimen that previously included valproic acid. Topiramate also has several proposed mechanisms for predisposing patients for hyperammonemia. There are limited data on topiramate monotherapy or long-term use of topiramate use causing hyperammonemia-associated encephalopathy or enhancing hyperammonemia with the addition of valproic acid. Conclusions: This case supports topiramate-related hyperammonemia and the pharmacodynamic interaction with the co-administration of valproic acid.
\end{abstract}

\section{Keywords}

topiramate, valproic acid, hyperammonemia, drug interaction, adverse reaction

\section{Introduction}

Topiramate is an antiepileptic indicated for the management of epilepsy and migraines. ${ }^{1}$ There are several proposed mechanisms for its activity, including blocking the release of sodium from neuronal voltage-gated sodium channels, enhancement of GABA receptors, antagonism of AMPA/kainite glutamate receptors, and inhibition of carbonic anhydrase. ${ }^{1}$ Hyperammonemia with a stupor encephalopathy has been associated with the use of topiramate primarily when topiramate is added to valproate antiepileptic regimens. ${ }^{2}$ Nausea, vomiting, weight loss, asterixis, confusion, somnolence, and coma are signs and symptoms of hyperammonemia. ${ }^{2}$

Hyperammonemia is defined as an elevated blood ammonia level with an unknown prognosis. ${ }^{3}$ Patients can present with irritability, headache, visual disturbances, seizures, or even coma. Not all patients with hyperammonemia will present with symptoms. Hyperammonemia is primarily caused by liver disorders and specifically associated with fulminant hepatic failure and hepatic encephalopathy. ${ }^{4}$ Other nonhepatic causes of hyperammonemia include, but not limited to, are inherited defects of the urea cycle enzymes, medications such chemotherapy, transport defect of intermediates in the urea cycle, parenteral nutrition, or urinary tract infections. ${ }^{5}$
Hyperammonemia is a well-established cause of encephalopathy. The suspected mechanism of ammonia affecting the brain is because the brain lacks an effective urea cycle. In the brain, glutamine formation is essential for the removal of ammonia. The astrocytes in the brain are responsible for glutamine formation, but in the presence of hyperammonia, cerebral edema occurs because of the accumulation of glutamine. This glutamine accumulation in the astrocytes causes an increase in the intracellular fluid of the astrocytes that causes them to swell. Another suspected mechanism of brain impairment is that the ammonium ion affects both the inhibitory and excitatory neurotransmitters causing an impaired movement of amino acids and monoamines. ${ }^{3}$

Topiramate has both pharmacokinetic and pharmacodynamic mechanisms linked to causing hyperammonemic encephalopathy. $^{2}$ The suspected mechanism for topiramate's cause of hyperammonemic encephalopathy is by

'Hospital of Special Surgery, New York, NY, USA

${ }^{2}$ Rutgers, The State University of New Jersey, Piscataway, NJ, USA

${ }^{3}$ Robert Wood Johnson University Hospital, New Brunswick, NJ, USA

Corresponding Author:

Kelly Moore, PharmD, Hospital of Special Surgery, 535 E 70th St,

New York, NY I002I, USA.

Email: Kelly.moore@wagner.edu 
indirectly inhibiting the production carbonyl-phosphate synthetase 1 (CPS-1), the first enzyme in the urea cycle. Topiramate inhibits carbonic anhydrase, which directly inhibits bicarbonate synthesis. The inhibition of bicarbonate results in reduced production of CPS-1. Topiramate also reduces the cerebral glutamine synthetase, which results in the potential increase in glutamate accumulation and brain edema. It has been suggested that topiramate possibly inhibits mitochondrial carbonic anhydrase, which would lead to the inhibition of the urea cycle and increase the ammonia. $^{3}$ The suspected mechanism from previous trials implies that topiramate synergistically enhances the mechanism of valproic acid and causes the hyperammonemia. ${ }^{4,5}$

Furthermore, our patient case illustrates the use of topiramate can enhance the potential for hyperammonemia with the addition of valproic acid.

\section{Case Report}

A 56-year-old female $(152.4 \mathrm{~cm} ; 46.4 \mathrm{~kg})$ with a past medical history of asthma, diverticulitis, arthritis, fibromyalgia, gastroesophageal reflux disease, migraines, depression, and restless leg syndrome presented to the emergency department following a severe asthma attack and subsequently was unresponsive and pulseless. In the field, the patient received chest compressions and several doses of her albuterol inhaler prior to emergency medical services arrival. The patient was defibrillated and given one dose of epinephrine en route to the hospital. On arrival, she was started on the code chill protocol, which is the utilization of therapeutic hypothermia in patients who have survived sudden cardiac death, and admitted to the cardiac care unit. The patient's home medications included methadone $10 \mathrm{mg}$ by mouth twice daily, albuterol inhaler 2 puffs by mouth 4 times a day as needed, fluticasone/salmeterol $250 \mathrm{mg} / 50 \mu \mathrm{g}$ 1 puff inhaled twice daily, hydromorphone $4 \mathrm{mg} 1$ to 2 tablets by mouth every 6 hours as needed, temazpam $30 \mathrm{mg}$ by mouth at bedtime, duloxetine $60 \mathrm{mg}$ by mouth daily, levetiracetam $1500 \mathrm{mg}$ by mouth twice daily for the chronic pain, eletriptan $40 \mathrm{mg}$ by mouth as needed for migraine, zolmitriptan nasal spray $5 \mathrm{mg}$ nasally as needed for migraine, topamax $100 \mathrm{mg}$ by mouth at bedtime for migraine prophylaxis, trazadone $100 \mathrm{mg}$ by mouth at bedtime, rabeprazole 20 $\mathrm{mg}$ by mouth daily, and trimethobenzamide $250 \mathrm{mg}$ by mouth every 8 hours as needed for nausea. The patient's topiramate, levetiracetam, and albuterol were continued on admission. After the rewarming of the patient posttherapeutic hypothermia, the patient was started on valproic acid (VPA) $750 \mathrm{mg}$ injection every 8 hours for the development of myoclonic seizures refractory to midazolam boluses. The first ammonia level was obtained 1 day after the start of the intravenous VPA. The ammonia level was elevated at $101 \mu \mathrm{g} / \mathrm{dL}$ and continued to rise over the next few days to a peak level of $265 \mu \mathrm{g} /$ $\mathrm{dL}$. The patient was then slowly titrated off topiramate and VPA was titrated down (see Table 1). The ammonia level was redrawn 6 days later and was $53 \mu \mathrm{g} / \mathrm{dL}$. Unfortunately, the patient was sedated and intubated during this course of her hospital stay, which made it difficult to assess the patient's resolution of symptoms of encephalopathy. The video electroencephalogram taken during the patient's hospital stay was suggestive of hypoxic encephalopathy due to cardiac arrest. Although the patient was encephalopatic, it cannot be determined if the hyperammonemia played a role. The patient remained unresponsive throughout her hospital stay, which limited the assessment of the patient.

The calculated Naranjo probability ${ }^{6}$ was 5 for a probable topiramate-induced hyperammonemia. See Table 1 for the patient's dosing and level during her hospital stay.

\section{Discussion}

Valproic acid-induced hyperammonemic encephalopathy is well documented. ${ }^{7-10}$ VPA has several alleged mechanisms of hyperammonemia encephalopathy. In the absence of hepatic dysfunction, VPA can cause a decrease in the hepatic urea synthesis. VPA causes an increase in ammonia production in the kidneys by its effects on decreasing the synthesis of glutamine, inhibition of carbymol phosphate synthase, and by reduction of hepatic ammonia metabolism by decrement of carnitine availability. Hyperammonemic encephalopathy occurs when patient's urea cycle decreases and when the ammonia level in the brain exceeds the patient's individual threshold, which results in a decreased state of consciousness. ${ }^{10}$

Previously case reports emphasized that the combination of both mechanisms can cause an increased chance of patients developing hyperammonemic encephalopathy. One study showed that the addition of topiramate to a VPA regimen increased VPA-induced encephalopathy from $0.13 \%$ to $0.57 \%$. ${ }^{9}$ The suspected mechanism from previous trials implies that topiramate synergistically enhances the mechanism of VPA and causes the hyperammonemia.

Currently in the literature case reports of VPA- and topiramate-induced hyperammonemia encephalopathies were associated with the addition of topiramate to a patient's medication regimen that previously included VPA. ${ }^{2}$ There are limited data on previous topiramate usage or long-term use of topiramate potentially enhancing the interaction with VPA. Deutsch and colleagues illustrated that the addition of VPA to a patient with bipolar disorder, borderline personality disorder, and migraine headaches already taking topiramate potentially caused hypermmonemic encephalopathy. ${ }^{11}$ This particular patient became symptomatic shortly after the addition of VPA and resolved 1 day after the diagnosis and discontinuation of VPA. ${ }^{11}$ Our patient was treated with topiramate and levetiracetam prior to arrival to the hospital, and patient had an elevated ammonia level following the coadministration of topiramate and VPA. When our patient's topiramate was stopped and VPA was tapered, the ammonia level returned to the normal range, thus supporting the interaction. We suspect that the previous use of topiramate and 
Table I. Timeline of Medication Administration and pertinent Laboratory Values ${ }^{\mathrm{a}}$.

\begin{tabular}{|c|c|c|c|c|c|}
\hline Day & Topiramate Dose & Valproic Acid Dose & $\begin{array}{c}\text { Ammonia Level } \\
(\mu \mathrm{g} / \mathrm{dL}) \text { (Reference } \\
\text { Range = 27-83) }\end{array}$ & $\begin{array}{l}\text { Valproic Acid Level } \\
(\mu g / \mathrm{mL})(\text { Reference } \\
\text { Range }=50-100)\end{array}$ & $\begin{array}{l}\text { Topiramate Level } \\
(\mu g / \mathrm{mL}) \text { (Reference } \\
\text { Range = 2-20) }\end{array}$ \\
\hline I & $100 \mathrm{mg}$ once daily & No dose & & & \\
\hline 2 & $100 \mathrm{mg}$ once daily & $\begin{array}{l}1500 \mathrm{mg} \text { in the morning; } \\
750 \mathrm{mg} \text { in the evening }\end{array}$ & & & \\
\hline 3 & 100 mg once daily & 750 mg 3 times a day & 101 & 81.5 & \\
\hline 4 & 100 mg twice daily & $750 \mathrm{mg} 3$ times a day & 140 & & \\
\hline 5 & 100 mg twice daily & $750 \mathrm{mg} 3$ times a day & 122 & & \\
\hline 6 & 100 mg twice daily & 750 mg 3 times a day & 271 & & \\
\hline 7 & 100 mg twice daily & $\begin{array}{l}750 \mathrm{mg} \text { in the morning; } \\
500 \mathrm{mg} \text { in the evening }\end{array}$ & 265 & 67.3 & 4.5 \\
\hline 8 & 100 mg twice daily & $500 \mathrm{mg}$ twice daily & & & \\
\hline 9 & 100 mg twice daily & 500 mg twice daily & & & \\
\hline 10 & 100 mg twice daily & 500 mg twice daily & 208 & 22.4 & \\
\hline II & $\begin{array}{l}50 \mathrm{mg} \text { once in the morning; } \\
100 \mathrm{mg} \text { in the evening }\end{array}$ & 500 mg twice daily & & & \\
\hline 12 & $50 \mathrm{mg}$ twice daily & $500 \mathrm{mg}$ twice daily & & & \\
\hline 13 & 50 mg once daily & 500 mg twice daily & & & \\
\hline $14-19$ & Stopped & 500 mg twice daily & & & \\
\hline 20 & & 500 mg twice daily & 53 & 42 & \\
\hline
\end{tabular}

${ }^{a}$ All laboratory values reflect the same day turnaround time.

the interaction with VPA caused our patient to have hyperammonemia.

Some limitations for this case include our patient was sedated throughout the time period of evaluation, and it is unclear if the patient's inability to be aroused can be attributed to hyperammonemia or to the sedation. The patient's encephalopathy could not be associated with topiramate or VPA because the patient was admitted with a hypoxic encephalopathy. Another limitation was that the baseline ammonia level was available and the elevated serum ammonia level could not be solely attributed to topiramate but rather the interaction between topiramate and VPA.

\section{Conclusion}

Topiramate was associated with hyperammonemia when administered in conjunction with valproic acid.

\section{Declaration of Conflicting Interests}

The author(s) declared no potential conflicts of interest with respect to the research, authorship, and/or publication of this article.

\section{Funding}

The author(s) received no financial support for the research, authorship, and/or publication of this article.

\section{References}

1. Topamax [Package insert]. Titusville, NJ: Janssen Pharmaceuticals, Inc; 2009.
2. Latour P, Biraben A, Polard E, et al. Drug induced encephalopathy in six epileptic patients: topiramate? valproate? or both? Hum Psychopharmacol. 2004;19:193-203.

3. Mathias R, Kostiner D, Packman S. Hyperammonemia in urea cycle disorders: role of the nephrologist. Am J Kidney Dis. 2001;37:1069-1080.

4. Brusilow SW. Hyperammonemic encephalopathy. Medicine (Baltimore). 2002;81:240-249.

5. Hawkes ND, Thomas GAO, Jurewicz A, et al. Non-hepatic hyperammonemia: an important potentially reversible cause of encephalopathy. Postgrad Med J. 2001;77:717-722.

6. Naranjo CA, Busto U, Sellers EM, et al. A method for estimating the probability of adverse drug reactions. Clin Pharmacol Ther. 1981;30:239-245.

7. Hamer HM, Knake S, Schomburg U, Rosenow F. Valproateinduced hyperammonemic encephalopathy in the presence of topiramate. Neurology. 2000;54:230-232.

8. Blackford MG, Do ST, Enlow TC, Reed MD. Valproic acid and topiramate induced hyperammonemic encephalopathy in a patient with normal serum carnitine. J Pediatr Pharmacol Ther. 2013;18:128-136.

9. Gomez-Ibañez A, Urrestarazu-Bolumburu E, Viteri-Torres C. Hyperammonemic encephalopathy related to valproate, phenobarbital, and topiramate synergism. Epilepsy Behav. 2011;21:480-482.

10. Noh Y, Kim D, Chu K, et al. Topiramate increase the risk of valproic acid-induced encephalopathy. Epilepsia. 2013;54: e1-e4.

11. Deutsch SI, Burket JA, Rosse RB. Valproate-induced hyperammonemic encephalopathy and normal liver functions: possible synergism with topiramate. Clin Neuropharmacol. 2009;32:350-352. 